OPEN ACCESS

Edited by:

Parvin Mehdipour:

Tehran University of Medical

Sciences, Iran

Reviewed by:

Weilin Pu,

Fudan University, China

Tanja Kunej,

University of Ljubljana, Slovenia

*Correspondence:

Xiaoyun Mao

maoxiaoyun@126.com

Specialty section:

This article was submitted to

Cancer Genetics,

a section of the journal

Frontiers in Oncology

Received: 03 July 2020

Accepted: 08 September 2020

Published: 20 October 2020

Citation:

Guo Y, Mao X, Qiao Z, Chen B and Jin $F$ (2020) A Novel Promoter CpG-Based Signature for Long-Term Survival Prediction of Breast Cancer

Patients. Front. Oncol. 10:579692.

doi: 10.3389/fonc.2020.579692

\section{A Novel Promoter CpG-Based Signature for Long-Term Survival Prediction of Breast Cancer Patients}

\author{
Yang Guo, Xiaoyun Mao*, Zhen Qiao, Bo Chen and Feng Jin \\ Department of Breast Surgery, The First Affiliated Hospital of China Medical University, Shenyang, China
}

DNA methylation has been reported as one of the most critical epigenetic aberrations during the tumorigenesis and development of breast cancer (BC). This study explored a novel promoter CpG-based signature for long-term survival prediction of BC patients. We used The Cancer Genome Atlas (TCGA) data as training set, and results were validated in an independent dataset from Gene Expression Omnibus (GEO). First, the differential methylation CpG sites were screened in TCGA dataset, of which the candidate promoter CpG sites were preliminarily identified with the univariate Cox regression analysis and the least absolute shrinkage and selection operator regression analysis. Second, the signature was constructed with stepwise regression analysis and multivariate Cox proportional hazards model, which was validated with the survival analysis of two cohorts each from TCGA and GEO databases. The 10-year receiver operating characteristic curves of risk score presented an area under the curve of over 0.7 for both cohorts. A nomogram was also constructed and released. Moreover, Gene Set Enrichment Analysis was performed to identify the more active pathways in high-risk patients. The CpG sites-target gene correlations and differential methylation regions were further explored. In conclusion, the promoter CpG-based signature exhibited good prognostic prediction efficacy in the long-term overall survival of BC patients.

Keywords: DNA methylation, breast cancer, prognosis, overall-survival, signature

\section{INTRODUCTION}

Breast cancer (BC) has become one of the most concerned public health issues in the worldwide, because of the growing incidence, high mortality, and huge economic burden $(1,2)$. More than 1 million new BC cases were diagnosed in 2002 (3). For women, BC led to $<25 \%$ of the newly diagnosed cancer cases and caused $14.7 \%$ of cancer-associated deaths (4). Further, the treatment costs of BC have been generally escalated with the advance of disease stage at diagnosis (5). BC patients can greatly benefit from early diagnosis, both in therapeutic efficacy and economic burden.

With the advances of molecular diagnosis technology, the heterogeneity and complexity of BC have been revealed (6). BC can be classified into different subgroups based on histopathologic characteristics or gene expression profiles. The molecular characterization of $\mathrm{BC}$ would provide much information for understanding the pathogenesis of $\mathrm{BC}$ and exploring potential markers for early diagnosis and target therapy (7).

DNA methylation, mainly occurring at cytosine-phosphate-guanosine dinucleotide (also designated as $\mathrm{CpG}$ ) where cytosine was converted to 5-methylcytosine (5meC), has been considered to make important effects in cancer development (8). The covalent addition of a 
methyl group was generally observed in cytosine within $\mathrm{CpG}$ dinucleotides, which were concentrated in large clusters called CpG islands (9). The abnormal methylation of promoter CpG dinucleotide sites in cancer leads to transcriptional silencing, which would be heritable in progeny cells (10). Because the DNA methylation can be varied with internal and external factors, it has become a research hotspot for investigating the tumorigenesis and cancer development (11). At the same time, DNA methylation has also been intensively explored as a target for epigenetic treatment.

Aberrant DNA methylation makes effects in BC. One study investigated the link between DNA methylation and gene expression in BC. The result identified a transcriptional network regulated by DNA methylation at enhancers, in a cell lineage-specific manner (12). Some BC-associated heritable DNA methylation markers were screened through detecting and comparing DNA methylation levels in BC patients and their family members (13). The endogenous and external factors may make effects via modulating DNA methylation patterns, such as oxidative DNA damage and age-related reproductive factors $(14,15)$.

After verifying the association between DNA methylation and gene expression in $\mathrm{BC}$, some large-scale studies have been performed to comprehensively explore the potential DNA methylation markers with clinical significance (16). In another study, blood-based DNA methylation biomarkers were summarized, which showed significance in risk stratification or the early detection of $\mathrm{BC}$ (17).

Infinium HumanMethylation 450 Bead Chip contained a total of $485,764 \mathrm{CpG}$ dinucleotide sites in the human genome. The whole genome could be divided into four regions: promoter, body, $3^{\prime}$-UTR ( $3^{\prime}$-untranslated region) and intergenic region. The promoter region was subdivided into $5^{\prime}$-UTR $\left(5^{\prime}\right.$ untranslated region), TSS200 (within $200 \mathrm{bp}$ upstream of the transcription start site), TSS1500 (within 1,500 bp upstream of the transcription start site), and 1stExon. According to the distance from $\mathrm{CpG}$ islands, $\mathrm{CpG}$ shores referred to the area within $2 \mathrm{~kb}$ upstream and downstream of $\mathrm{CpG}$ islands; $\mathrm{CpG}$ shelves referred to the area within $2 \mathrm{~kb}$ upstream and downstream of $\mathrm{CpG}$ shores; open sea referred to other areas except $\mathrm{CpG}$ islands, $\mathrm{CpG}$ shores, and $\mathrm{CpG}$ shelves. From the functional genome distribution standpoint, 200,339 CpG sites were located in promoter regions; 15,383, 150,212 and $119,830 \mathrm{CpG}$ sites correspond to $3^{\prime}$-UTR, gene body, and intergenic-open sea sequences, respectively. From the $\mathrm{CpG}$ content and neighborhood context, 150,254 were located in CpG islands, 112,072 in CpG shores, 47,161 in CpG shelves, and 176,277 were isolated CpG sites in the genome defined as "open sea" (18).

In this study, we aimed to explore novel survival-associated promoter $\mathrm{CpG}$ dinucleotide sites for prognostic prediction in BC patients. The BC methylation $450 \mathrm{~K}$ datasets and corresponding clinical features were obtained from both The Cancer Genome Atlas (TCGA) and Gene Expression Omnibus (GEO) databases. First, the differential methylation $\mathrm{CpG}$ $(\mathrm{dmCpG})$ sites were screened in TCGA dataset, of which the candidate promoter $\mathrm{CpG}$ sites were preliminarily identified with the univariate Cox regression analysis and the least absolute shrinkage and selection operator (LASSO) regression analysis. Second, the promoter CpG-based signature was constructed with stepwise regression analysis, which was validated with the survival analysis of two cohorts each from TCGA and GEO databases. A nomogram was also constructed, and the calibration curves showed that it was able to predict 5-, 7-, and 10-year survival accurately. Moreover, Gene Set Enrichment Analysis (GSEA) was performed to identify the more active pathways in high-risk patients and the CpG sites-target gene correlations and differential methylation regions (DMRs) were further explored.

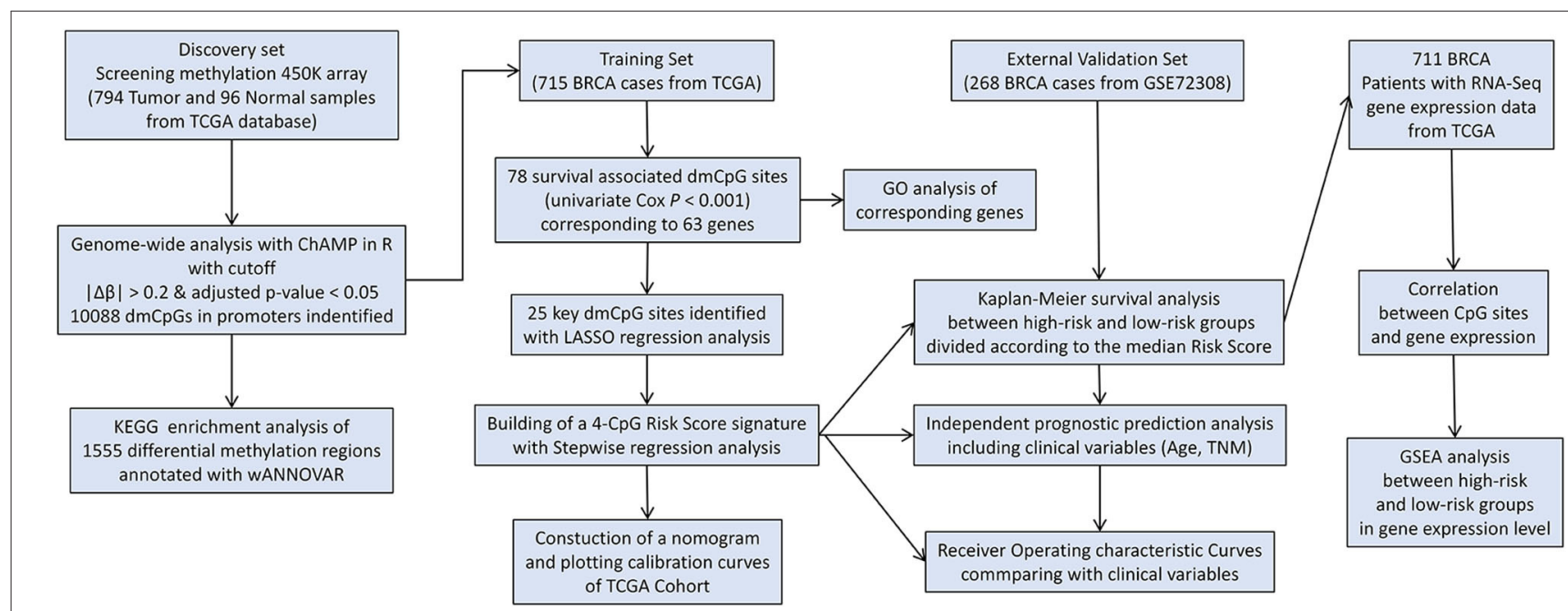

SCHEME 1 | Flow diagram of the analysis procedure: data collection, processing, analysis, and validation. 
A

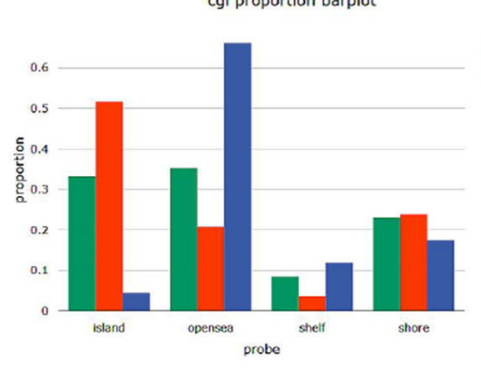

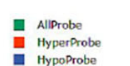

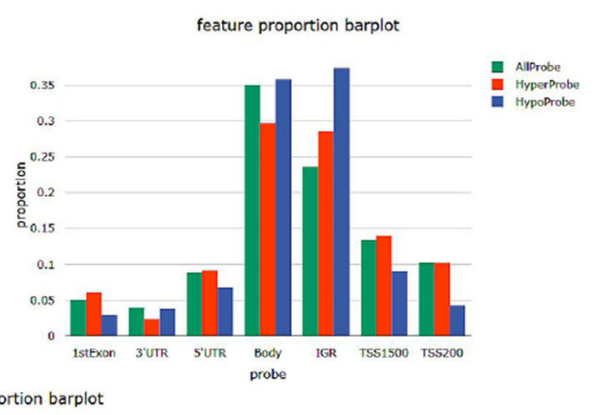

feature-cgi proportion barplot

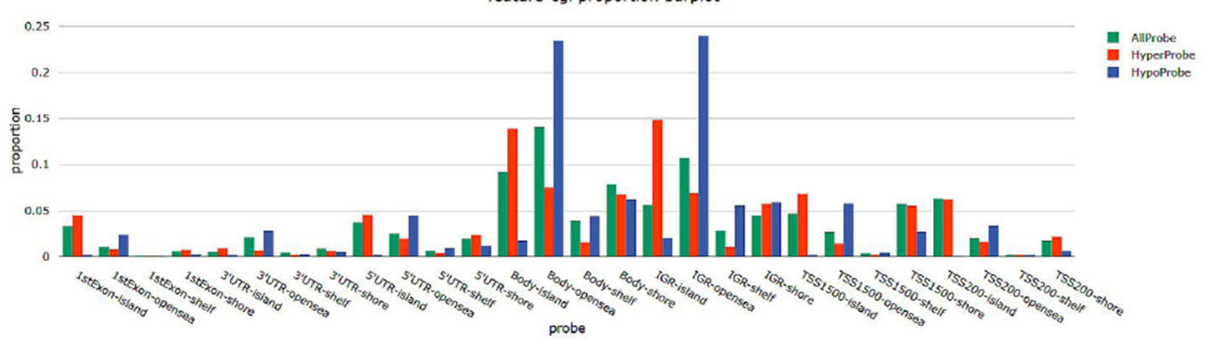

B

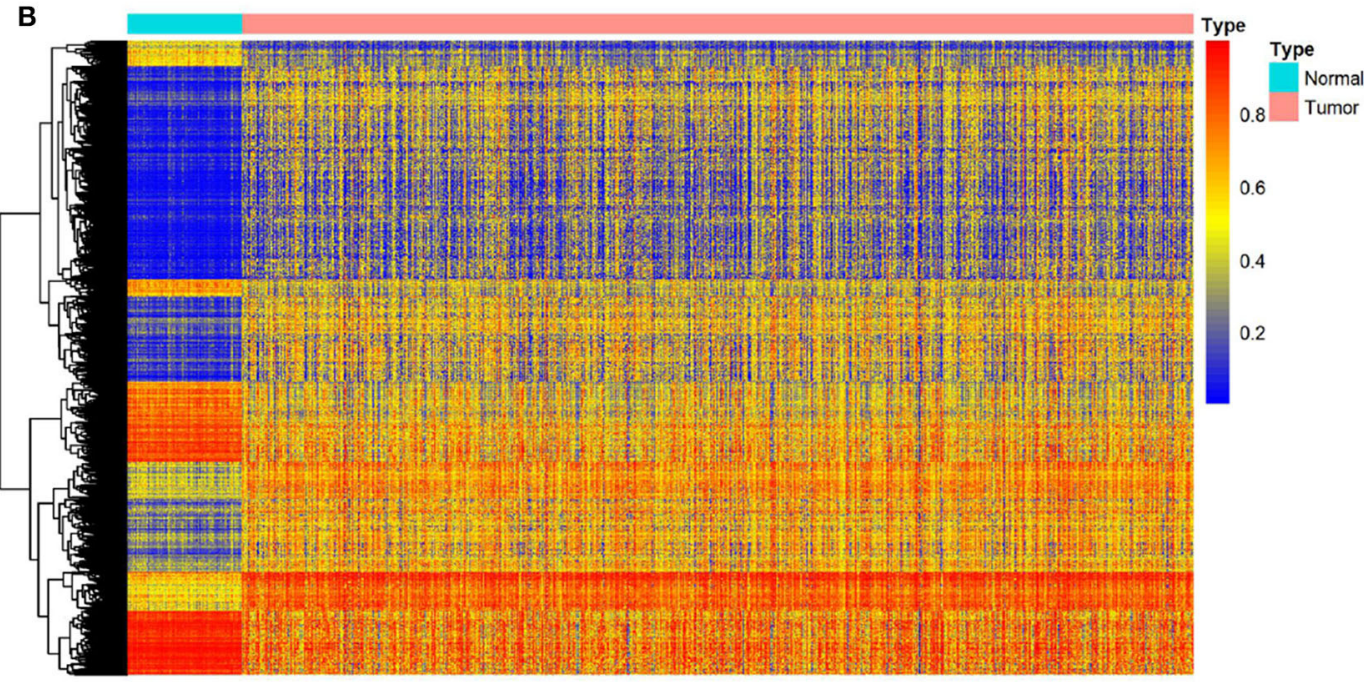

C

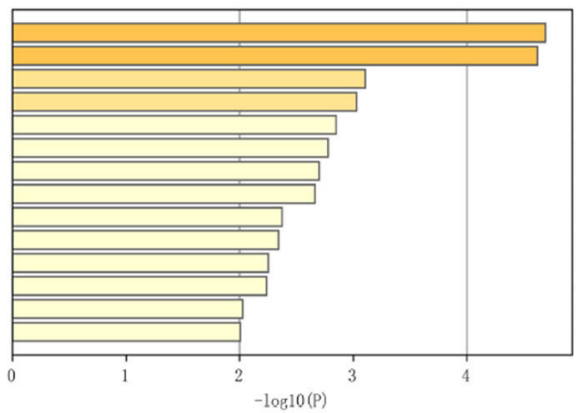

G0:0009952: anterior/posterior pattern specification

G0:0015874: norepinephrine transport

G0:0030522: intracellular receptor signaling pathray

G0:0048732: gland development

60:1905475: regulation of protein localization to membrane

60:0010506: regulation of autophagy

G0: 1990823: response to leukemia inhibitory factor

60:0070997: neuron death

60:0045936: negative regulation of phosphate metabolic process

hsa04728: Dopaninergic synapse

60:0060041: retina development in camera-type eye

60:0007411: axon guidance

60:0032680: regulation of tumor necrosis factor production

60:0043433: negative regulation of DNA-binding transcription factor activity

$-\log 10(P)$

FIGURE 1 | Identification and function enrichment of dmCpG sites in the TCGA cohort. (A) Histogram of the distribution regions of dmCpG sites in the genome. (B) Heatmap of dmCpG sites in the promoter region between normal and tumor samples. (C) GO function enrichment analysis of 78 dmCpG sites. 
TABLE 1 | The univariate Cox regression analysis of TCGA cohort.

\begin{tabular}{|c|c|c|c|c|c|c|c|c|c|c|c|}
\hline CpG ID & HR & HR.95L & HR.95H & $p$ & Gene & CpG ID & HR & HR.95L & HR.95H & $p$ & Gene \\
\hline cg03225817 & 9.135 & 3.827 & 21.807 & 0.000 & GRIA4 & cg17253709 & 0.031 & 0.005 & 0.214 & 0.000 & B3GNT2 \\
\hline cg07972135 & 9.093 & 3.774 & 21.909 & 0.000 & GRIA4 & cg13525197 & 6.825 & 2.349 & 19.830 & 0.000 & ZSCAN23 \\
\hline cg25482786 & 9.768 & 3.481 & 27.410 & 0.000 & $\mathrm{CHL} 1$ & cg01321962 & 0.274 & 0.134 & 0.563 & 0.000 & ESR1 \\
\hline cg09093993 & 0.123 & 0.047 & 0.319 & 0.000 & PLEKHF2 & cg18784113 & 0.143 & 0.049 & 0.424 & 0.000 & THRB \\
\hline cg09869811 & 0.115 & 0.042 & 0.317 & 0.000 & NUTF2 & cg15379185 & 5.720 & 2.159 & 15.150 & 0.000 & $E V X 1$ \\
\hline cg03940684 & 5.322 & 2.397 & 11.817 & 0.000 & $\mathrm{CHL} 1$ & cg03329976 & 8.503 & 2.568 & 28.162 & 0.000 & SOX17 \\
\hline cg07746943 & 6.081 & 2.570 & 14.392 & 0.000 & $\mathrm{CHL1}$ & cg24970620 & 0.166 & 0.061 & 0.454 & 0.000 & SLC7A7 \\
\hline cg06332621 & 0.110 & 0.038 & 0.325 & 0.000 & RBM47 & cg19002907 & 0.187 & 0.073 & 0.478 & 0.000 & TDRD1 \\
\hline cg21217024 & 4.660 & 2.169 & 10.011 & 0.000 & GRIA4 & cg11429283 & 0.208 & 0.086 & 0.501 & 0.000 & SYTL2 \\
\hline cg02631468 & 8.651 & 2.959 & 25.291 & 0.000 & $V S \times 1$ & cg04975519 & 0.189 & 0.074 & 0.481 & 0.000 & RNF32 \\
\hline cg06818710 & 6.783 & 2.596 & 17.727 & 0.000 & ZSCAN23 & cg20924470 & 4.494 & 1.930 & 10.465 & 0.000 & FBXL21 \\
\hline cg00043788 & 8.475 & 2.896 & 24.800 & 0.000 & $V S \times 1$ & cg02006107 & 0.164 & 0.059 & 0.454 & 0.001 & MRPS28 \\
\hline cg17576603 & 0.155 & 0.060 & 0.398 & 0.000 & $D A B 2$ & cg16703956 & 6.069 & 2.187 & 16.842 & 0.001 & SLC6A3 \\
\hline cg01279654 & 0.193 & 0.084 & 0.444 & 0.000 & VGLL4 & cg27626299 & 7.201 & 2.347 & 22.088 & 0.001 & $E V X 1$ \\
\hline cg02919712 & 0.109 & 0.035 & 0.338 & 0.000 & DEPDC6 & cg10898212 & 0.167 & 0.061 & 0.463 & 0.001 & $C D K 5 R 1$ \\
\hline cg23559689 & 4.498 & 2.075 & 9.749 & 0.000 & GRIA4 & cg05144884 & 0.210 & 0.086 & 0.511 & 0.001 & PRSS27 \\
\hline cg25609507 & 5.104 & 2.203 & 11.822 & 0.000 & CHAT & cg14763548 & 3.816 & 1.780 & 8.184 & 0.001 & $V S X 1$ \\
\hline cg25160286 & 7.081 & 2.578 & 19.454 & 0.000 & $E V \times 1$ & cg09529093 & 0.215 & 0.089 & 0.519 & 0.001 & ANGPT1 \\
\hline cg15287850 & 0.089 & 0.025 & 0.310 & 0.000 & ST6GAL1 & cg26065909 & 0.111 & 0.031 & 0.391 & 0.001 & SELENBP1 \\
\hline cg09884146 & 0.047 & 0.010 & 0.230 & 0.000 & SPRED2 & cg04034767 & 3.489 & 1.703 & 7.151 & 0.001 & GRASP \\
\hline cg25520146 & 0.190 & 0.080 & 0.452 & 0.000 & HPYR1 & cg06327814 & 0.159 & 0.055 & 0.457 & 0.001 & C7orf53 \\
\hline cg04054313 & 6.529 & 2.410 & 17.687 & 0.000 & GLT1D1 & cg13027458 & 0.181 & 0.068 & 0.483 & 0.001 & LOC644649 \\
\hline cg10546487 & 0.115 & 0.036 & 0.365 & 0.000 & CLIC4 & cg12042659 & 3.690 & 1.738 & 7.831 & 0.001 & ZNF132 \\
\hline cg05481991 & 5.512 & 2.217 & 13.704 & 0.000 & HMGA2 & cg08506163 & 6.358 & 2.187 & 18.485 & 0.001 & C6orf174 \\
\hline cg08539965 & 0.105 & 0.032 & 0.350 & 0.000 & EIF4G3 & cg02337653 & 5.839 & 2.108 & 16.175 & 0.001 & C6orf174 \\
\hline cg09968620 & 6.839 & 2.445 & 19.128 & 0.000 & HOXD9 & cg05223720 & 4.847 & 1.943 & 12.091 & 0.001 & CALN1 \\
\hline cg10603275 & 4.181 & 1.943 & 8.997 & 0.000 & $\mathrm{CHL1}$ & cg05863502 & 9.087 & 2.519 & 32.783 & 0.001 & CACNA1B \\
\hline cg00822495 & 4.572 & 2.020 & 10.349 & 0.000 & OTX2 & cg11523712 & 5.754 & 2.073 & 15.973 & 0.001 & HOXD13 \\
\hline cg09260773 & 6.071 & 2.303 & 16.005 & 0.000 & $T B \times 15$ & cg27304204 & 0.240 & 0.104 & 0.555 & 0.001 & TSPAN8 \\
\hline cg05663573 & 6.106 & 2.297 & 16.237 & 0.000 & SLC7A14 & cg26657920 & 0.203 & 0.079 & 0.517 & 0.001 & ACTBL2 \\
\hline cg09871043 & 0.065 & 0.015 & 0.285 & 0.000 & PKHD1 & cg16636226 & 0.239 & 0.103 & 0.553 & 0.001 & LRRC3B \\
\hline cg13557668 & 5.153 & 2.117 & 12.545 & 0.000 & FBXL21 & cg14768785 & 4.648 & 1.886 & 11.453 & 0.001 & GHSR \\
\hline cg06884401 & 0.121 & 0.039 & 0.381 & 0.000 & FAM13A & cg17465304 & 0.126 & 0.038 & 0.426 & 0.001 & KIF12 \\
\hline cg16716750 & 4.143 & 1.915 & 8.963 & 0.000 & RGS17 & cg24475782 & 5.650 & 2.032 & 15.705 & 0.001 & TBX15 \\
\hline cg23458558 & 5.638 & 2.190 & 14.516 & 0.000 & $R A L Y L$ & cg06578434 & 0.146 & 0.047 & 0.455 & 0.001 & SBNO2 \\
\hline cg07277549 & 0.176 & 0.068 & 0.459 & 0.000 & NOD1 & cg14650610 & 3.463 & 1.661 & 7.222 & 0.001 & SPOCK1 \\
\hline cg11413039 & 6.424 & 2.301 & 17.934 & 0.000 & PUS3 & cg00930873 & 5.258 & 1.969 & 14.046 & 0.001 & $A L D H 1 A 2$ \\
\hline cg18397523 & 6.636 & 2.331 & 18.887 & 0.000 & $E V \times 1$ & cg00880452 & 5.975 & 2.071 & 17.244 & 0.001 & SYCN \\
\hline cg01035160 & 4.861 & 2.027 & 11.659 & 0.000 & SNCA & cg13032463 & 4.145 & 1.782 & 9.640 & 0.001 & $T$ \\
\hline
\end{tabular}

\section{MATERIALS AND METHODS Data Resources}

Two datasets were included in this analysis. Breast-invasive carcinoma methylation $450 \mathrm{~K}$ dataset (designated as TCGABRCA) and clinical information of patients were downloaded from UCSC Xena database (https://xena.ucsc.edu/), involving 96 normal samples and 794 tumor samples. The dataset GSE72308 and corresponding clinical information were downloaded from the GEO database (designated as GEO-BRCA), including 295 patients (19). All the data were obtained based on the platform of Illumina Infinium Human Methylation 450 Bead Chip.

\section{Data Filter and Normalization}

For the two datasets, the methylation beta matrix was filtered and normalized with ChAMP R package (20). The batch effect was removed with SVA R package. Probes in two datasets were filtered with the exclusion criteria including: (i) detection $p>0.01$; (ii) bead count $<3$ in at least $5 \%$ of samples; (iii) non-CpG sites; 

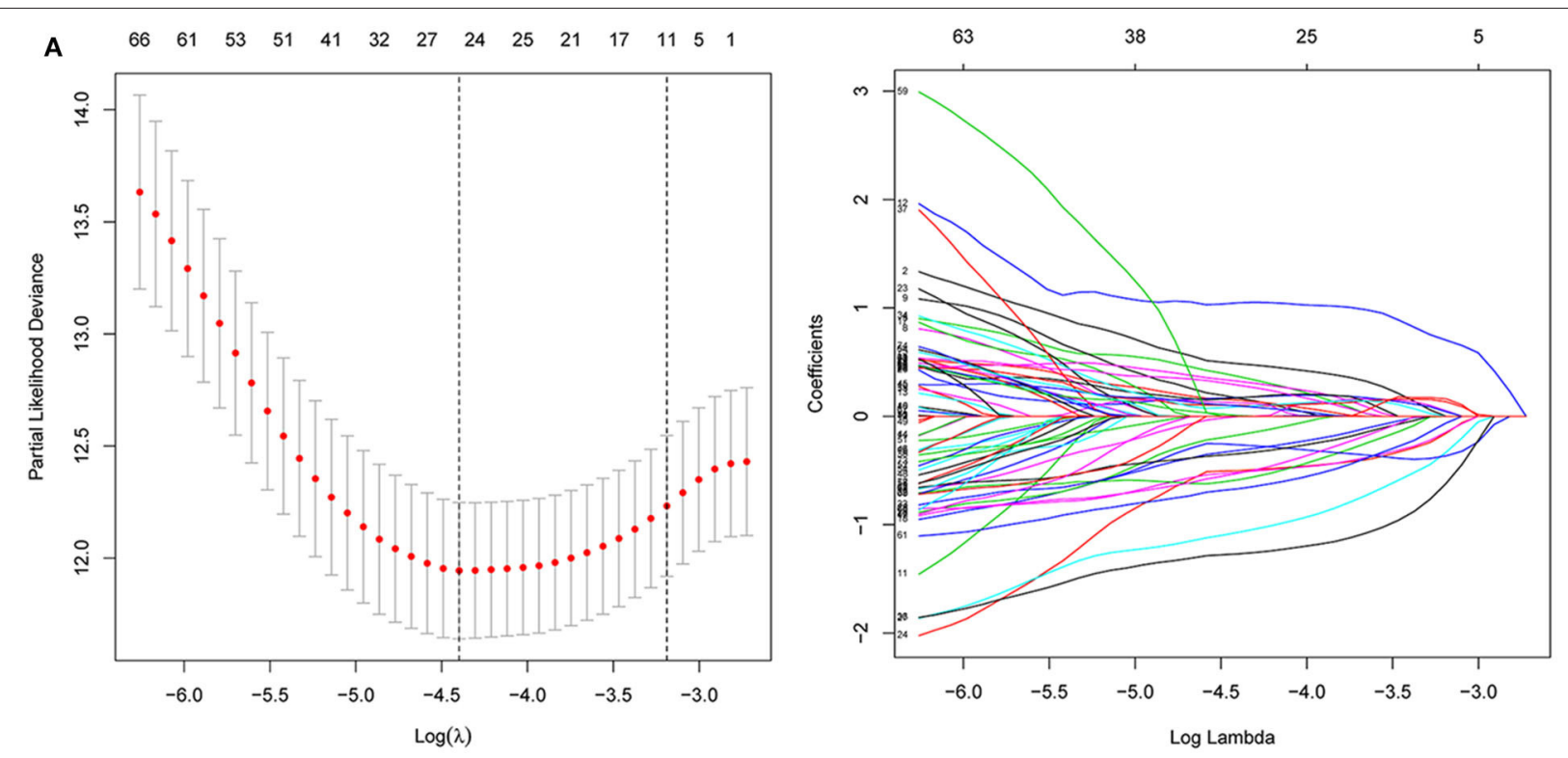

B
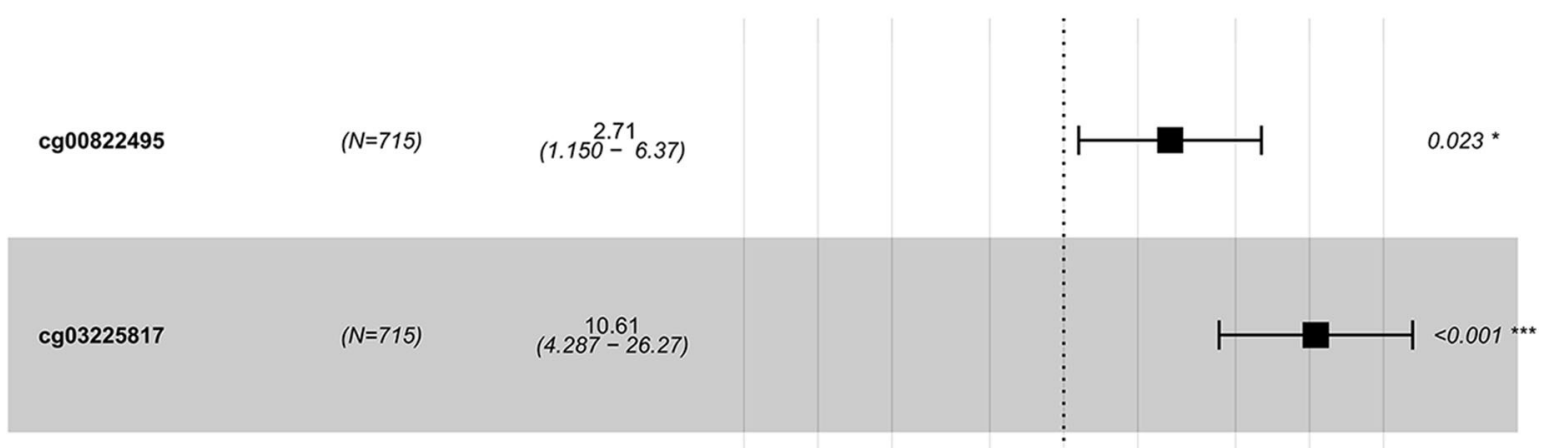

cg06884401

$(N=715)$

$(0.036-0.29)$

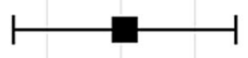

$<0.001^{\star * *}$
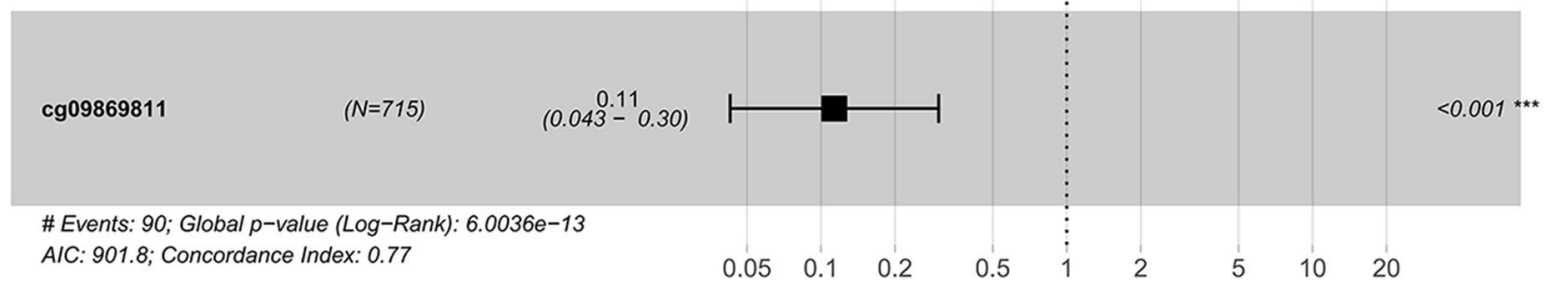

FIGURE 2 | The construction of dmCpG-based prognostic signature. (A) LASSO regression analysis of CpG sites in the regions of promoter, with the selection of tuning parameter (lambda) and dynamic LASSO coefficient profiling. (B) The HR and coefficient of each dmCpG involved in the multivariate Cox proportional hazards model.

(iv) all SNP-related probes and multihit probes; and (v) probes on X and Y chromosomes. The BMIQ method was applied for types I and II probe correction. In particular, probes with SNPs were identified in general $450 \mathrm{~K}$ SNP list (21) and filtered by champ.filter() function included in ChAMP R package.

\section{dmCpG Sites}

The $\mathrm{dmCpG}$ sites were preliminarily screened with ChAMP $\mathrm{R}$ package. In this study, CpG sites with $|\Delta \beta|>0.2$ and BenjaminiHochberg adjusted $P<0.05$ were identified as $\mathrm{dmCpG}$ sites. The
$\mathrm{dmCpG}$ sites located in the promoter regions ( $5^{\prime}$-UTR, TSS200, TSS1500 and 1stExon) were further screened and visualized with the pheatmap R package.

\section{The dmCpG Sites Identification in TCGA Dataset}

TCGA dataset was applied as training cohort, of which only the patients with overall survival (OS) ranging from 90 to 3,650 days were included for further analysis. The males and patients without OS information were removed, and 715 

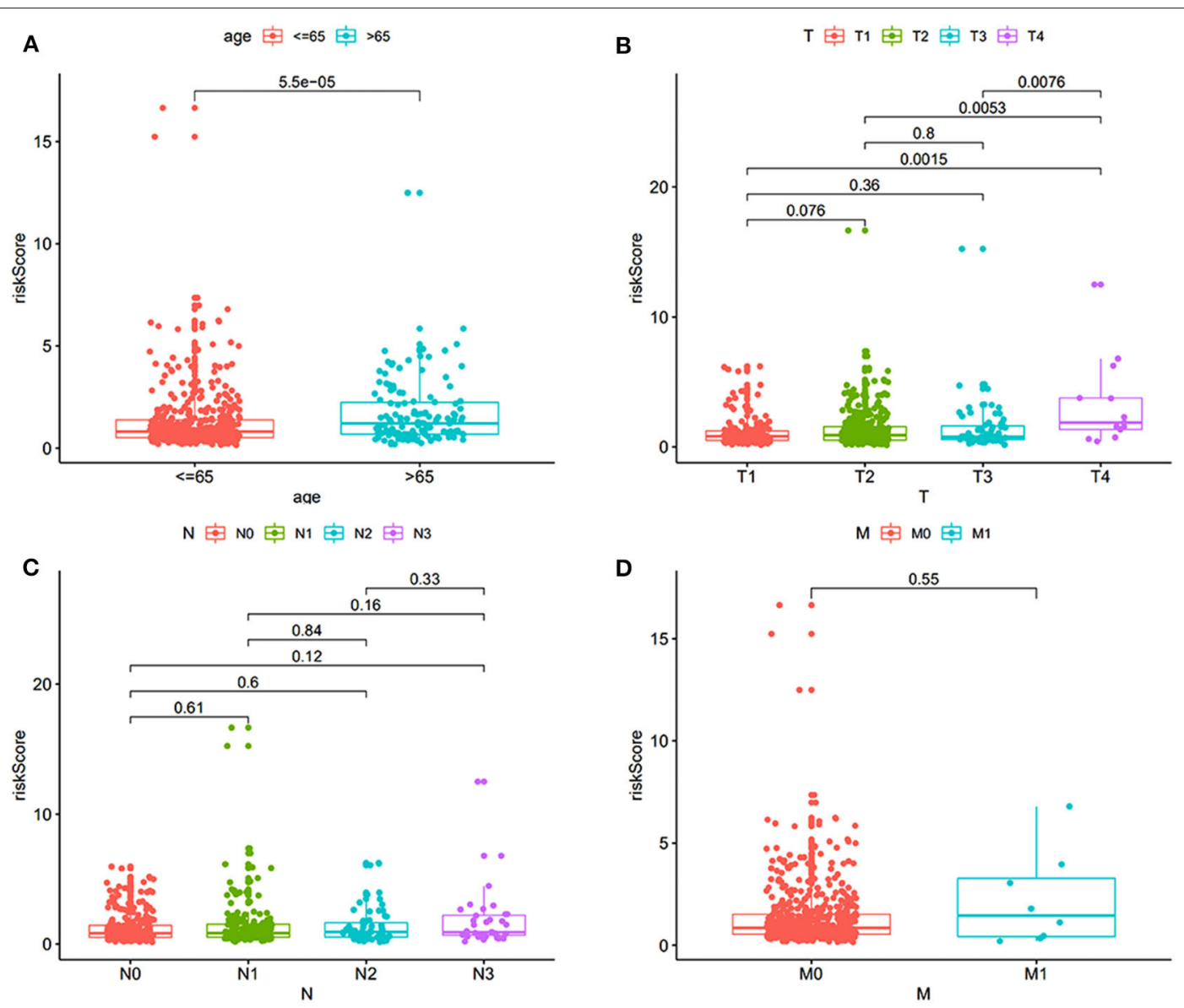

FIGURE 3 | The correlation of risk score and clinical features, including age and TNM (tumor, node, metastasis). (A) Correlation with age. (B) Correlation with T stage. (C) Correlation with different lymph node status. (D) Correlation with distant metastasis.

patients were finally included. The univariate Cox regression analysis was applied for screening OS-associated $\mathrm{dmCpG}$ sites preliminarily with survival $\mathrm{R}$ package. The hazard ratio (HR) and $p$-value were provided. The $\mathrm{dmCpG}$ sites with $P<$ 0.001 and $\mathrm{HR}<10$ were screened. A total of 78 survivalassociated $\mathrm{dmCpG}$ sites were obtained, corresponding to 63 genes. The metascape (www.metascape.org) was applied for Gene Ontology (GO) function enrichment analysis (22). LASSO regression analysis was further performed to explore the key dmCpG sites with glmnet R package. Finally, $25 \mathrm{dmCpG}$ sites were identified.

\section{The Construction of CpG-Based Prognostic Signature}

With the identified 25 OS-associated $\mathrm{dmCpG}$ sites, stepwise regression analysis was applied to optimize the model. Variables with $P<0.01$ in the stepwise analysis were included in a multivariate Cox proportional hazards model. Under the optimal situations, the dmCpG sites and corresponding coefficients were presented, and the formula for calculating the prognostic index (designated as risk score) based on methylation levels of $\mathrm{dmCpG}$ sites was obtained.

\section{The Relationship of Risk Score With Clinical Characteristics}

A total of 565 patients with complete clinical information in TCGA dataset were included. The ggpubr $\mathrm{R}$ package and $t$ test were involved to explore the relationships between the CpG-based risk score and clinical characteristics, including age and TNM (tumor, node, metastasis). $P<$ 0.05 indicates statistically significant. The results were provided with boxplots.

\section{Independent Prognostic Prediction Analysis}

Univariate and multivariate independent prognostic analyses were applied with survival $\mathrm{R}$ package. The included clinical variables were age, TNM (tumor, node, metastasis), and risk score. The 565 patients with complete clinical information in the TCGA dataset were included. The HR was calculated and expressed with forest plot. The 5-, 7-, and 10-year receiver operating characteristic (ROC) curves of risk score, age, and TNM (tumor, node, metastasis) were plotted with survival ROC $\mathrm{R}$ package. Area under the curve (AUC) of the ROC curve was also provided. 


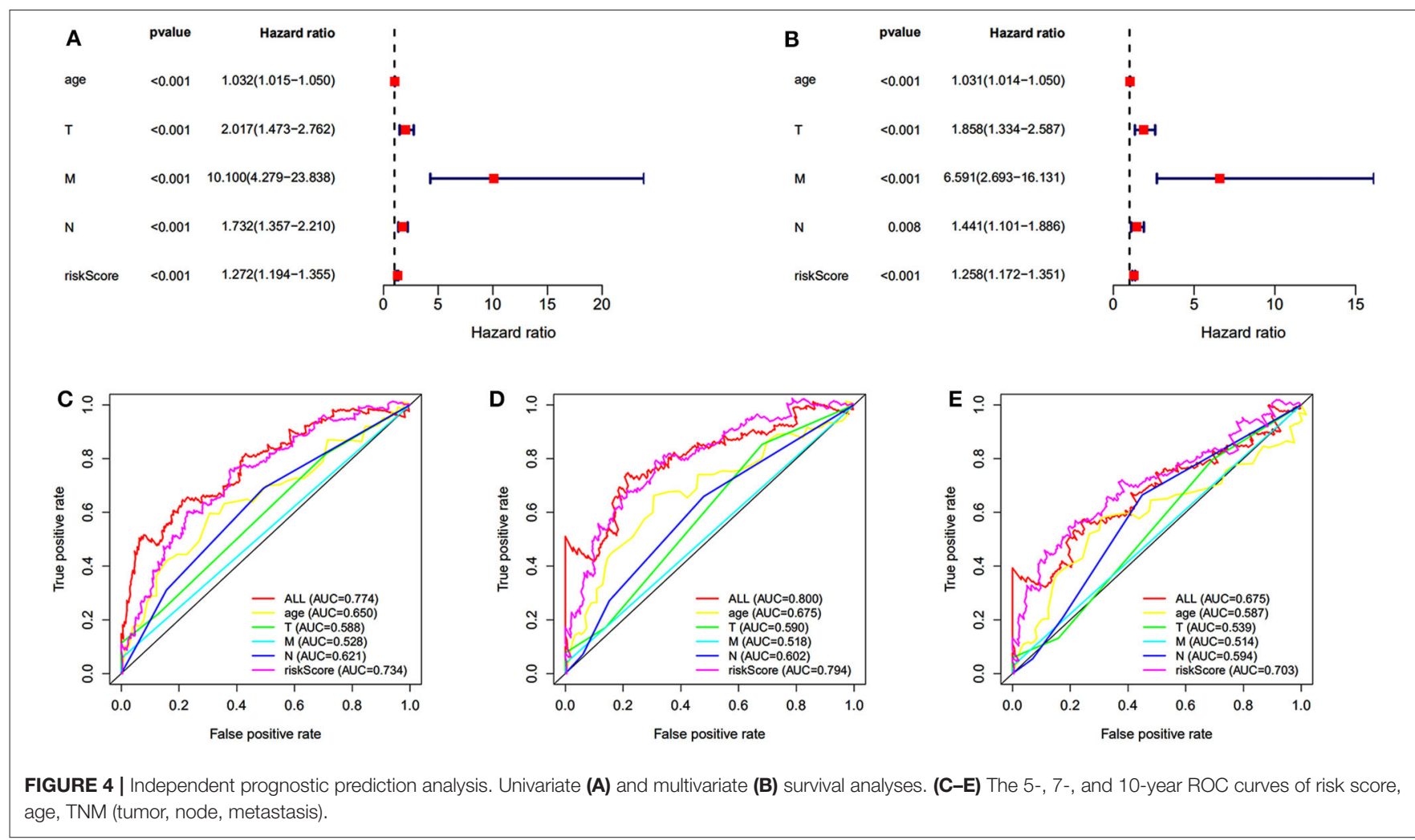

\section{The Validation of dmCpG-Based Signature}

The prognostic prediction efficacy of $\mathrm{dmCpG}$-based signature was validated in both TCGA and GEO datasets. The survival analysis was performed on 715 patients in TCGA dataset and 268 patients in GEO dataset, respectively, with survival $\mathrm{R}$ package. The risk score was calculated based on the methylation level of included $\mathrm{dmCpG}$ sites and corresponding coefficients. The patients could be classified as high- and lowrisk groups, with the median risk score in TCGA dataset as cutoff value. The survival analyses of patients in high- and low-risk groups were performed with survival $\mathrm{R}$ package. The 5-, 7-, and 10-year ROC curves were plotted, and AUC of the ROC curve was calculated. Further, the nomogram was constructed for the patients in TCGA dataset with rms R package, and the calibration curves were also plotted with calibrate function.

\section{Analysis of Included dmCpG Sites}

The dmCpG sites were further investigated. First, the correlation between $\mathrm{dmCpG}$ sites and located gene expression was explored. The RNA-Seq expression profile (FPKM) of TCGA-BRCA was downloaded from GDC Data Portal (https://portal.gdc.cancer. gov). The Pearson correlation coefficient between $\beta$ value of dmCpG sites and FPKM value of located genes was calculated with cor.test in $\mathrm{R}$ and visualized. Second, based on the transcriptome data, the GSEA was performed to identify the more active pathways in low-risk patients with GSEA 4.0.1 with 184 background gene sets in c2.cp.kegg.v6.2.symbols.gmt.
Finally, the DMRs were screened with ChAMP R package and annotated with wANNOVAR (http://wannovar.wglab.org/) (23). The KEGG pathway enrichment analysis was further performed with ConsensusPathDB (http://cpdb.molgen.mpg.de/) (24).

\section{RESULTS}

\section{The dmCpG Sites Identification}

The study flowchart describing the process is shown in Scheme $\mathbf{1 .}$ The datasets from TCGA and GEO databases were preprocessed for further comparison, including filter, normalization, and batch correction. Then the $\mathrm{dmCpG}$ sites were screened in TCGA dataset with cutoff of $|\Delta \beta|>0.2$ and adjusted $P<0.05$. The proportions of $\mathrm{dmCpG}$ sites were screened (Figure 1A), and a total of $10,088 \mathrm{dmCpG}$ sites located in the promoter regions (5'-UTR, TSS200, TSS1500, and 1stExon) were finally identified (Figure 1B).

Second, the univariate Cox regression analysis was performed to identify the candidate OS-associated $\mathrm{dmCpG}$ sites in TCGA cohort. A total of 78 survival-associated dmCpG sites with $P<0.001$ were identified, corresponding to 63 genes (Table 1). The GO function enrichment of the 63 genes indicated several GO terms, including anterior/posterior pattern specification, norepinephrine transport, intracellular receptor signaling pathway, gland development, regulation of autophagy, etc. (Figure 1C). LASSO regression analysis was further performed to explore the key dmCpG sites. Finally, 25 sites were identified (Figure 2A). 

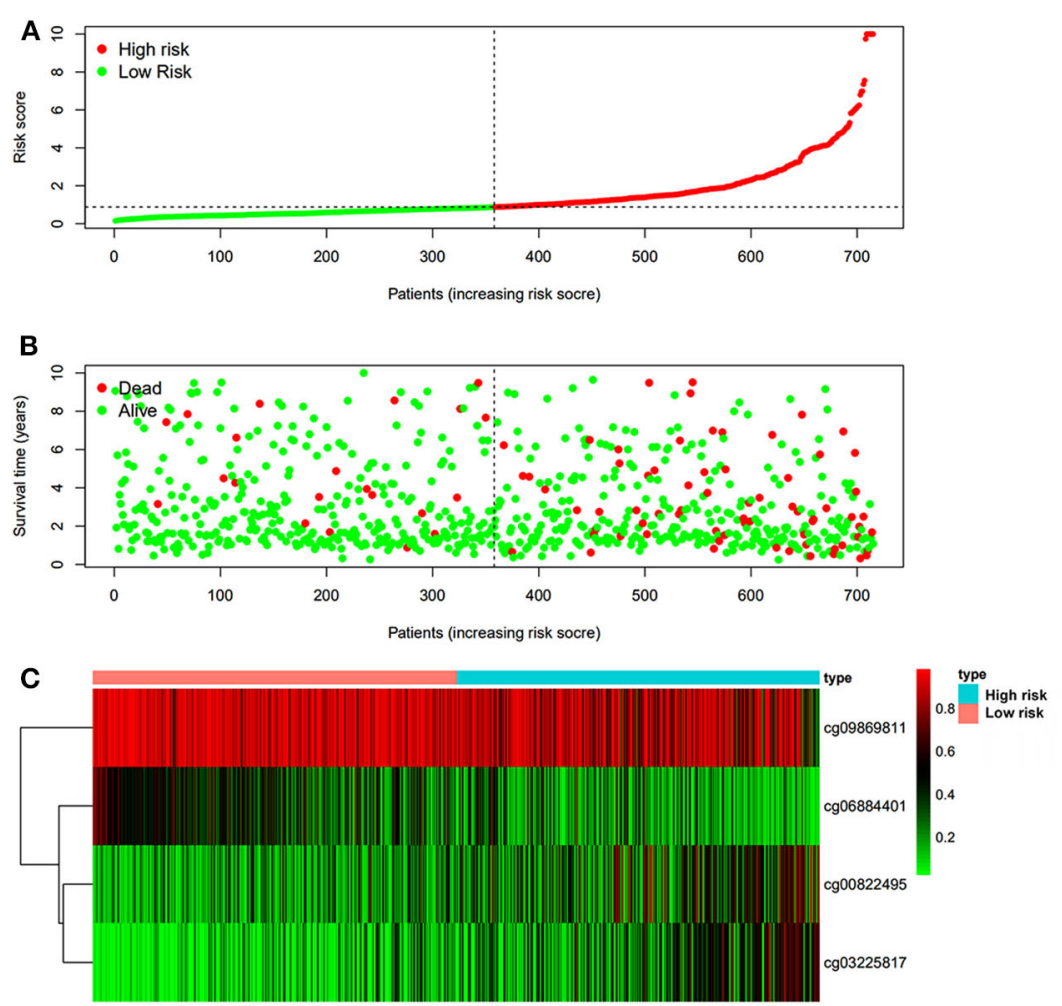
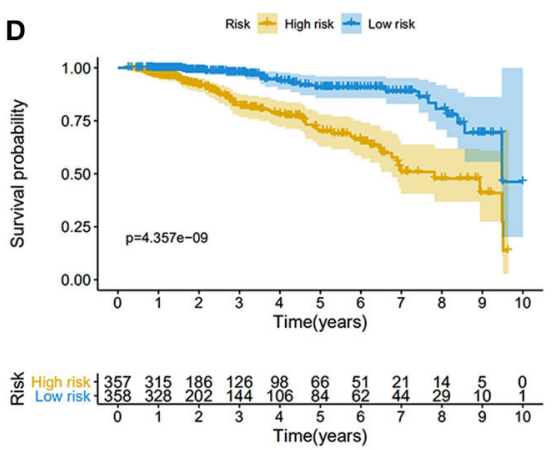

E ROC curve of TCGA corhort

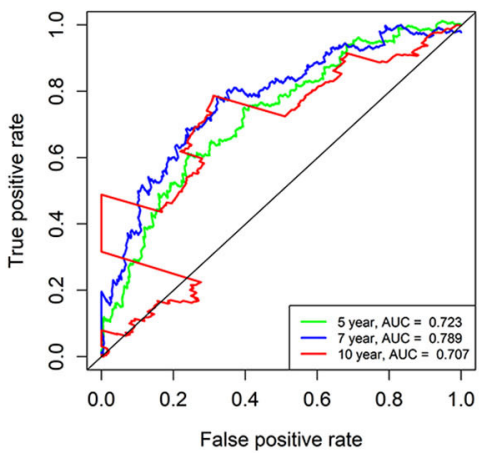

FIGURE 5 | Risk score in the TCGA cohorts. (A) The rank of calculated risk score. (B) The survival status of high- and low-risk patients. (C) Heatmap of methylation level of 4 CpG sites. (D) Kaplan-Meier survival curve of the patients classified in high- and low-risk groups. (E) The 5-, 7-, and 10-year ROC curves of risk score.

\section{The Construction of dmCpG-Based Prognostic Signature}

With the identified 25 survival-associated dmCpG sites, the stepwise regression analysis was further applied to screen the candidate $\mathrm{dmCpG}$ sites for constructing the prognostic signature (Figure 2B). Ten candidate $\mathrm{dmCpGs}$ sites were screened in the stepwise analysis (Supplementary Figure 1). Further, four variables with $P<0.01$ in the stepwise analysis were included in a multivariate Cox proportional hazards model. These four prognostic $\mathrm{dmCpG}$ sites were finally included in the model for calculating the risk score. In the optimal model, the AIC was 901.8, and concordance index was 0.77. The $\mathrm{HR}$ and coefficient of each $\mathrm{dmCpG}$ was provided (Figure 2C). The risk score was calculated with the following formula: risk score $=\operatorname{cg} 00822495 \times 0.9960+$ $\operatorname{cg} 03225817 \times 2.3621-\operatorname{cg} 06884401 \times 2.2685-\operatorname{cg} 09869811$ $\times 2.1771$.

\section{The Relationship of Risk Score With Clinical Characteristics}

A total of 565 patients with complete clinical information in TCGA dataset were included. The risk score was significantly higher in patients with older than 65 years (Figure 3A). Further, the risk score was significantly higher for patients at T4 compared to that of other $\mathrm{T}$ stages (Figure 3B). No differences were found in patients with different lymph node statuses and distant metastases (Figures 3C-D).

\section{Independent Prognostic Prediction Analysis}

Univariate and multivariate survival analyses were performed to evaluate whether the risk score was an independent prognostic index irrespective of other clinical features. The clinical information of 565 patients in TCGA dataset was included. The univariate and multivariate analyses indicated that the age, TNM (tumor, node, metastasis), and risk score were an independent prognostic index $(P<0.05$ for all, Figures 4A,B). The AUC of 5-, 7-, and 10-year ROC curves indicated that the risk score provided a higher value of AUC compared to that of other clinical features (Figures 4C-E). The risk score was verified as independent prognostic predictor. Further, the integration of risk score and clinical features provided similar AUC of 5-, 7-, and 10-year ROC curves compared to that of individual risk score, indicating comparable prognostic prediction efficacy.

\section{Prognostic Prediction Analysis}

The prognostic prediction analysis was performed in TCGA and GEO cohorts, respectively. For patients in the TCGA cohort, risk score was calculated according to the normalized 

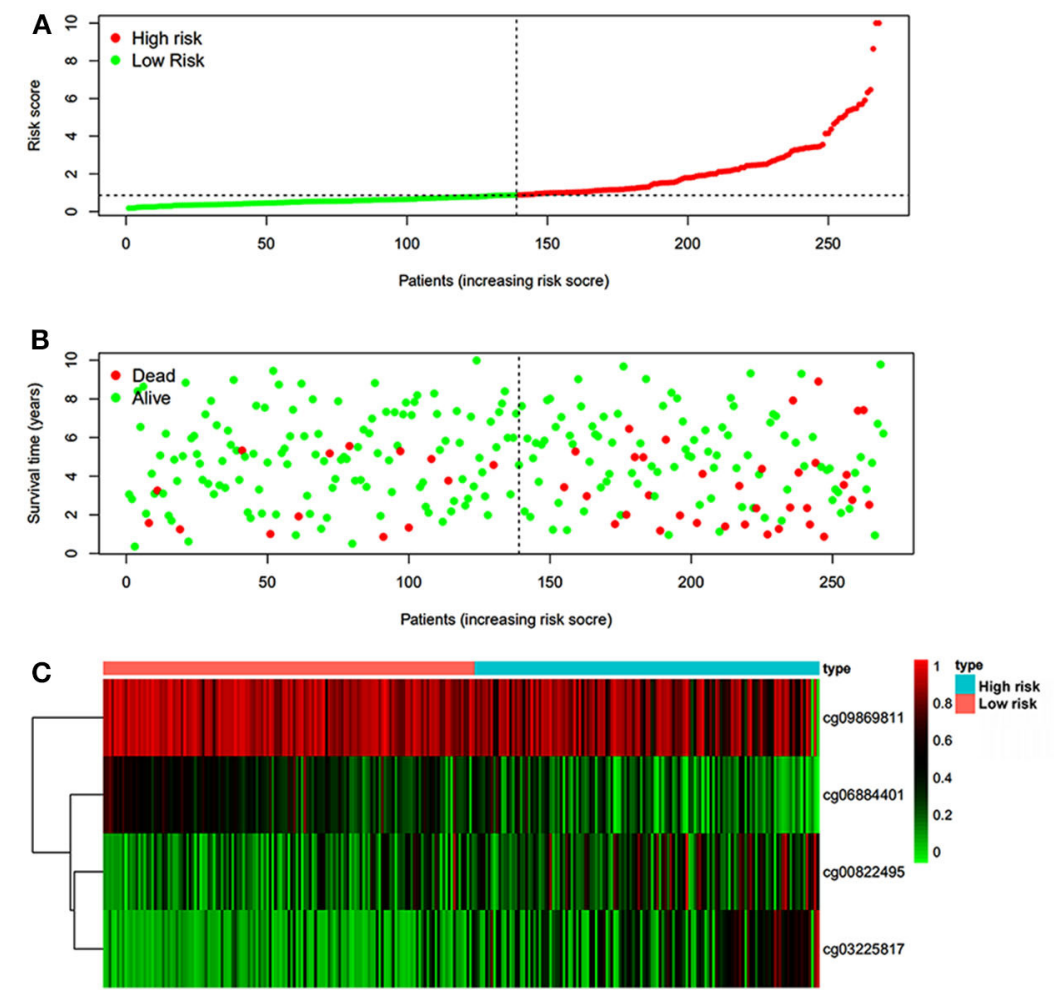
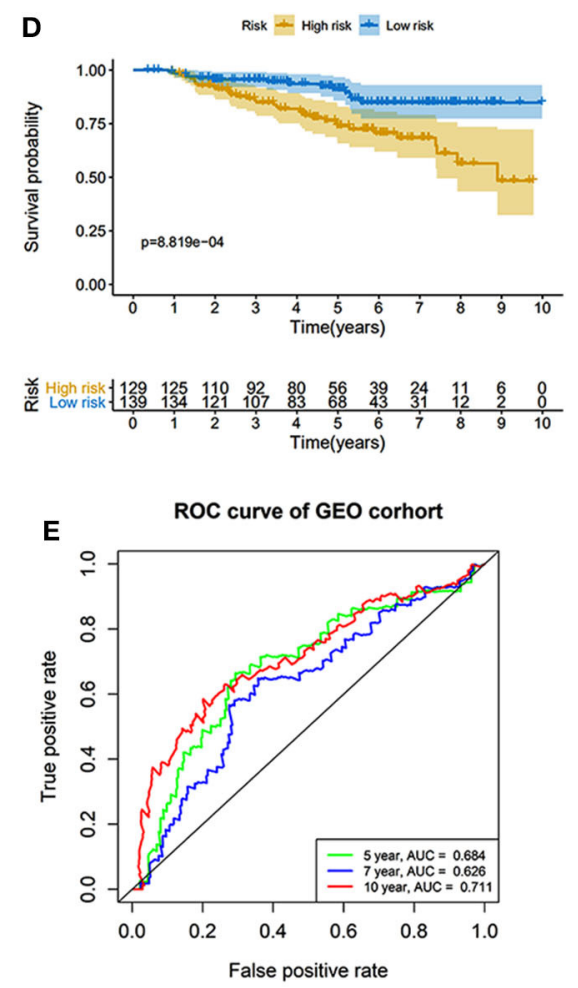

FIGURE 6 | Risk score in the GEO cohorts. (A) The rank of calculated risk score. (B) The survival status of high- and low-risk patients. (C) Heatmap of methylation level of 4 CpG sites. (D) Kaplan-Meier survival curve of the patients classified in high- and low-risk groups. (E) The 5-, 7-, and 10-year ROC curves of risk score.

methylation levels of four $\mathrm{dmCpG}$ sites. The median risk score of 0.8775 was applied as the cutoff for dividing patients into high- and low-risk groups (Figure 5A). The distribution of survival status of all patients was also presented (Figure 5B). The variation tendency of the methylation level of dmCpG sites in heatmap was consistent with their coefficients in prognostic signature (Figure 5C). Kaplan-Meier survival analysis of the risk score indicated the survival probability of patients in highand low-risk groups $(P<0.001$, Figure 5D). Further, 5-, 7-, and 10-year ROC curves of risk score were plotted, with the AUCs of $0.723,0.789$, and 0.707 , respectively (Figure 5E). The AUC value indicated good prognostic prediction efficacy. With the risk score of 0.8775 as cutoff, the prognostic prediction ability of risk score was also validated in the patients in the GEO cohort; similar results can be obtained (Figure 6). The 5-, 7-, and 10-year ROC curves of risk score were plotted, with the AUCs of $0.684,0.622$, and 0.711 , respectively (Figure 6E).

The nomogram was plotted for the TCGA cohort to calculate the risk score and predict 5-, 7-, and 10-year OS of BC patients (Figure 7A). The calibration curves were also provided (Figures 7B-D). The observed model presented with black solid line seemed close to ideal prediction model presented with blue dot and light gray line. The dynamic nomogram was released online at the following website: https://cpgsignature-survival-breastcancer.shinyapps.io/ cpgsignature-survival-breastcancer/.

\section{Analysis of Included CpG Sites}

The identified dmCpG sites were analyzed for further exploring their function mechanism. First, the characteristics of involved dmCpG sites were extracted, including located gene and regions (Table 2). Second, RNA-Seq data were obtained in 711 samples from TCGA cohort. The correlations between the four $\mathrm{dmCpG}$ sites in the signature and their target gene expression were analyzed (Figure 8A). Positive association was observed in cg00822495-OTX2 $(r=0.17, P=0.000)$, whereas negative association was observed between cg06884401FAM13A $(r=-0.28, P=0.000)$ and cg03225817-GRIA4 $(r=-0.12, P=0.000)$. No significant correlation was observed in cg09869811-NUTF2 $(r=-0.03, P=0.49)$ (Figure 8A). Third, GSEA was performed to identify the more active pathways in low-risk patients. Thirteen pathways were more obviously enriched, which were antigen processing and presentation, apoptosis, B-cell receptor signaling, cell adhesion molecules cams, chemokine signaling pathway, cytokine-cytokine receptor interaction, FC gamma $\mathrm{r}$-mediated phagocytosis, hematopoietic cell lineage, JAK-STAT signaling pathway, leukocyte transendothelial migration, natural killer cell-mediated cytotoxicity, T-cell receptor signaling pathway, and toll-like receptor signaling pathway (Figure 8B). Finally, DMRs were explored to further understand the function mechanism of methylation. A total of 1,555 screened DMRs were annotated, and the KEGG pathway enrichment analysis was performed. The 10 most enriched pathways were provided 


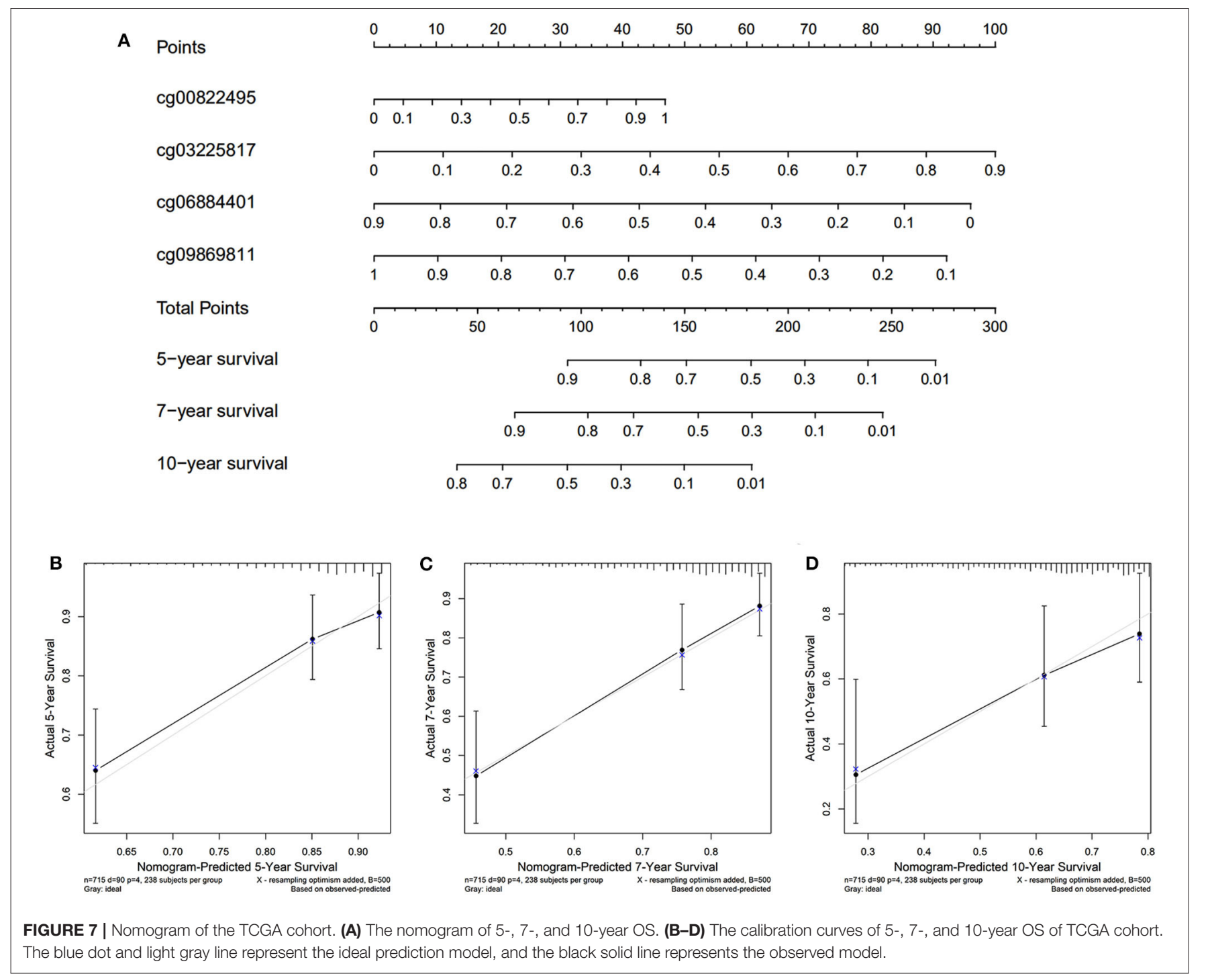

TABLE 2 | The characteristics of dmCpG sites.

\begin{tabular}{lllll}
\hline CpG ID & Gene & Feature & Ccgi & Feat.cgi \\
\hline cg00822495 & OTX2 & 5'-UTR & island & 5'-UTR-island \\
cg03225817 & GRIA4 & 5'-UTR & island & 5'-UTR-island \\
cg06884401 & FAM13A & 1stExon & opensea & 1stExon-opensea \\
cg09869811 & NUTF2 & 5'-UTR & opensea & 5'-UTR-opensea \\
\hline
\end{tabular}

(Figure 8C). In these pathways, PI3K-Akt signaling pathway showed most involved DMRs and frequent interactions with other terms.

\section{DISCUSSION}

The epigenetic aberrations have been observed during the tumorigenesis and development of $\operatorname{BC}(25,26)$. The DNA methylation located at gene promoter would generally suppress gene transcription, thus down-regulating the expression level of target genes. One study investigated DNA methylation with methylation quantitative trait loci of 30,477 CpG sites in 122,977 BC patients and 105,974 controls of European descent (16). They screened $199 \mathrm{CpG}$ sites with significant association with $\mathrm{BC}$ risk. Based on the methylation detection tools and computational analysis, the relationship between DNA methylation and gene expression can be well-demonstrated for identifying new biomarkers for BC (16). Considering the benefit of early and non-invasive diagnosis, blood sample-based DNA methylation was developed. One comprehensive study identified DNA methylation markers in blood for diagnosis or risk evaluation of BC. Variations in DNA methylation profiles, both at overall genomic level and specific loci, have been associated with BC risk (17). Another large scale meta-analysis identified genes consistently associated with prognosis, and their DNA methylation could indicate prognosis and clinical stratification of BC patients (27). 


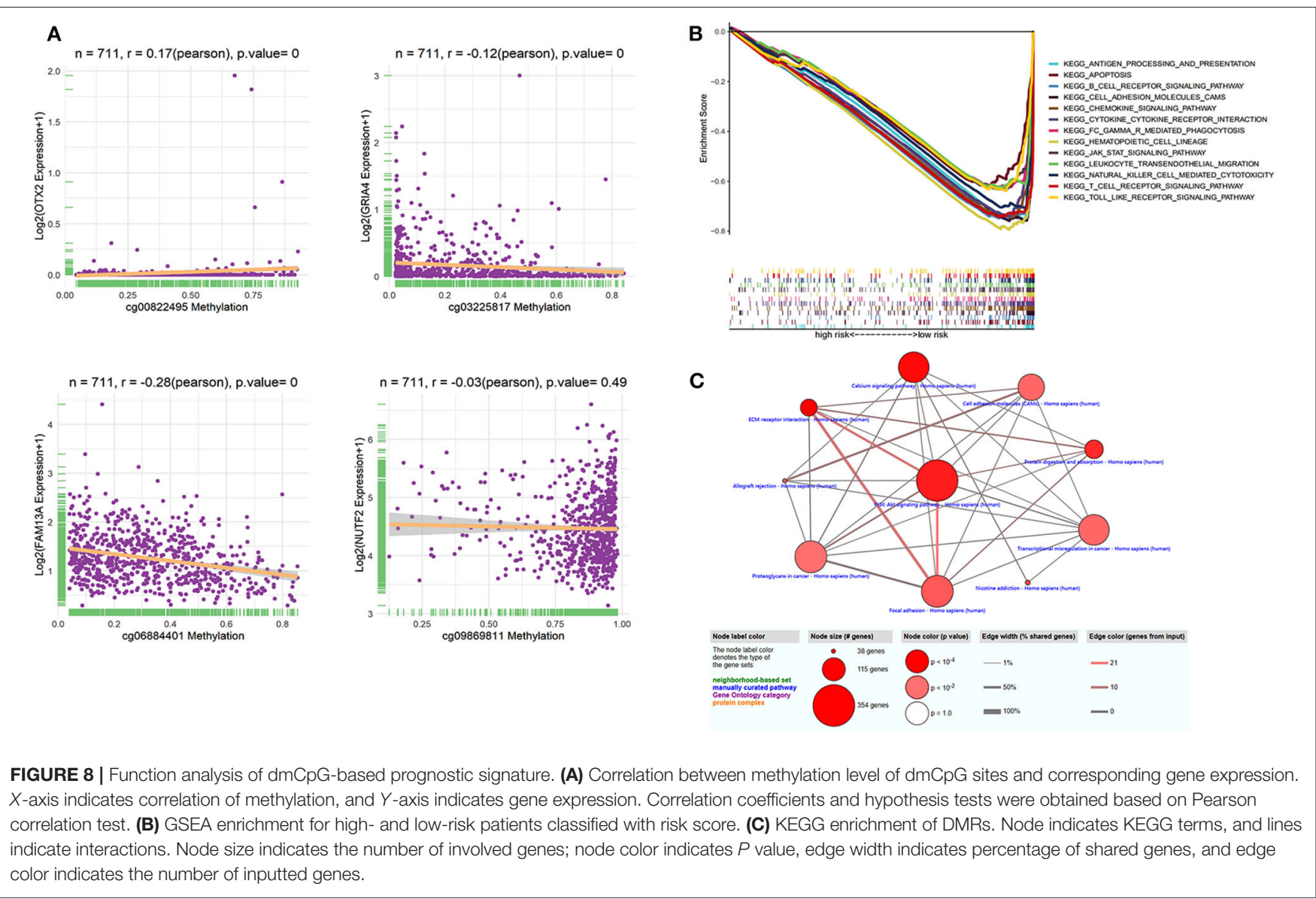

Some experimental evidence explored and validated the candidate DNA methylation sites. A fast and accurate methylation marker-based automated cartridge system was developed to detect $\mathrm{BC}$ in cells obtained by fine-needle aspiration. The panel consisted of 10 highly methylated markers, presenting an AUC over 0.9 in discriminating $\mathrm{BC}$ and benign breast lesions (28). Methylation signatures involving 100 or $30 \mathrm{CpG}$ probes were validated to discriminate patients with or without recurrence (29). Accumulating evidences proved the correlation between global DNA methylation and BC risk, which could be modified by reproductive characteristics (15), oxidative DNA damage (14), and chemotherapeutic agents (30).

In recent years, several prognostic prediction indexes have been developed, based on the expression levels of mRNA, lncRNAs, miRNAs, and so on. One study reported a seven-gene signature for prognostic prediction and treatment guidance in triple-negative BC (TNBC), in which recurrence risk score can be calculated as follows: $\mathrm{mRNA}$ signature $=1.108 \times$ TMEM101 - $0.213 \times$ KRT5 - $0.315 \times A C A N-0.464 \times L C A 5+0.446 \times$ $R P P 40-0.373 \times$ LAGE3 - $0.257 \times$ CDKL2 (31). More studies reported that the integrated IncRNA-mRNA signature provided better prognostic prediction efficacy. The developed response score involved 1 lncRNA and 2 coding genes: response score $=2.595 \times$ BPESC1 $-1.09 \times W D R 72-1.428 \times$ GADD $45 A$ - 0.731 (32). Another integrated mRNA-lncRNA signature was developed based on the mRNA species for FCGR1A, RSAD2, CHRDL1, and the IncRNA species for HIF1A-AS2 and AK124454, which may be applied to predict tumor recurrence and the benefit of taxane chemotherapy in TNBC (33). A study was performed to construct mRNA-only signature and integrated mRNA-lncRNA signatures. Both signatures provided similar results for prognostic prediction, while integrated signature had a higher hazard of recurrence (34). Another systematic analysis of lncRNA-miRNA-mRNA competing endogenous RNA network identified four-lncRNA signature as a prognostic biomarker for BC, which were ADAMTS9-AS1, LINC00536, AL391421.1, and LINC00491 (35).

DNA methylation was investigated as novel epigenetic biomarkers for prognostic prediction in $\mathrm{BC}$, because they can increase cancer risk through altering gene expression (36). A mortality risk score based on 10 selected CpGs exhibited strong association with all-cause mortality, cardiovascular diseases, and cancer mortality in BC (37). In our study, based on the in silico analysis, OS-associated promoter $\mathrm{CpG}$ sites signature was constructed for prognostic prediction of $\mathrm{BC}$, of which four $\mathrm{dmCpG}$ sites were identified, including cg00822495 (OTX2), cg03225817 (GRIA4), cg06884401 (FAM13A), and cg09869811 (NUTF2). In this signature, the higher methylation level of cg00822495 (OTX2) and cg03225817 (GRIA4) indicated higher risk of poor survival, while the higher methylation 
level of cg06884401 (FAM13A) and cg09869811 (NUTF2) indicated lower risk. Some of the involved target genes were previously reported. OTX2 has been considered as a significantly hypermethylated gene in BC (38). GRIA4 was a methylation-dependent gene involved in neural signaling (39). It was known as an early event in colorectal cancer carcinogenesis (40). One study has reported that rs1059122 (FAM13A) might result in BC susceptibility in Chinese population (41). FAM13A was also reported as a hypoxiainduced gene in non-small cell lung cancer, which was overexpressed under hypoxia conditions (42). NUTF2 was a novel methylation site for BC, which has not been previously reported.

Although the CpG-based signature explored in our study has a better performance than age and TNM stage, there were several limitations to be considered. First, it was only an in silico and retrospective study of publicly available data. The validation of the prediction was performed in only one independent cohort. Adequate validation in a larger population-based prospective cohort should be further performed to strengthen the clinical utility. Second, the integration of risk score with other previously reported markers such as age and TNM might enable the development of more reliable biomarkers. Further study would be necessary. Third, the biological functions of some related target genes should be explored and verified.

In recent years, with the rapid development of genomedetecting technology, we are entering an era of precise treatment. A lot of biomarkers based on gene expression profiles were identified, but very few were learned from CpG dinucleotide sites. The four-CpG signature and nomogram explored in our study could guide clinicians to predict long-term survival of BC patients, accurately identify high-risk patients, and take early intervention in the treatment. It is a fact that the detection of $\mathrm{CpG}$ sites is more complex and expensive than gene expression

\section{REFERENCES}

1. Coughlin SS, Ekwueme DU. Breast cancer as a global health concern. Cancer Epidemiol. (2009) 33:315-8. doi: 10.1016/j.canep.2009.10.003

2. Bray F, McCarron P, Parkin DM. The changing global patterns of female breast cancer incidence and mortality. Breast Cancer Res. (2004) 6:229. doi: 10.1186/bcr932

3. Ferlay J, Bray F, Pisani P, Parkin D. GLOBOCAN 2002. Cancer Incidence, Mortality and Prevalence Worldwide. IARC CancerBase No. 5. version 2.0. New York, NY: Springer (2002).

4. Ferlay J, Soerjomataram I, Dikshit R, Eser S, Mathers C, Rebelo M, et al. Cancer incidence and mortality worldwide: sources, methods and major patterns in GLOBOCAN 2012. Int J Cancer. (2015) 136:E359-86. doi: 10.1002/ijc. 29210

5. Sun L, Legood R, dos-Santos-Silva I, Gaiha SM, Sadique Z. Global treatment costs of breast cancer by stage: a systematic review. PLoS ONE. (2018) 13:e0207993. doi: 10.1371/journal.pone.0207993

6. Gatza ML, Lucas JE, Barry WT, Kim JW, Wang Q, Crawford MD, et al. A pathway-based classification of human breast cancer. Proc Natl Acad Sci USA. (2010) 107:6994-9. doi: 10.1073/pnas.0912708107

7. Allinen M, Beroukhim R, Cai L, Brennan C, Lahti-Domenici J, Huang H, et al. Molecular characterization of the tumor microenvironment in breast cancer. Cancer Cell. (2004) 6:17-32. doi: 10.1016/j.ccr.2004.06.010 detection now, but hundreds of thousands of CpG sites contain promising diagnostic and prognostic value, which would be explored with the development of detection technology in the future.

\section{CONCLUSIONS}

This study explored a novel promoter CpG-based signature that exhibited good prognostic prediction efficacy in the long-term OS of BC patients.

\section{DATA AVAILABILITY STATEMENT}

All datasets generated for this study are included in the article/Supplementary Material.

\section{AUTHOR CONTRIBUTIONS}

YG analyzed the data and wrote the manuscript. XM and ZQ helped to collect relevant papers for this research. BC analyzed the data and generated the figures and tables. FJ guided the research process. All authors read and approved the final manuscript.

\section{FUNDING}

The project was supported by Natural Science Fund of The First Affiliated Hospital of China Medical University.

\section{SUPPLEMENTARY MATERIAL}

The Supplementary Material for this article can be found online at: https://www.frontiersin.org/articles/10.3389/fonc. 2020.579692/full\#supplementary-material
8. Kulis M, Esteller M. DNA methylation and cancer. Adv Genet. (2010) 70:2756. doi: 10.1016/B978-0-12-380866-0.60002-2

9. Iacobuzio-Donahue CA. Epigenetic changes in cancer. Annu Rev Pathol. (2009) 4:229-49. doi: 10.1146/annurev.pathol.3.121806.151442

10. Wang Y-P, Lei Q-Y. Metabolic recoding of epigenetics in cancer. Cancer Commun. (2018) 38:25. doi: 10.1186/s40880-018-0302-3

11. Daura-Oller E, Cabre M, Montero MA, Paternain JL, Romeu A. Specific gene hypomethylation and cancer: new insights into coding region feature trends. Bioinformation. (2009) 3:340. doi: 10.6026/97320630003340

12. Fleischer T, Tekpli X, Mathelier A, Wang S, Nebdal D, Dhakal HP, et al. DNA methylation at enhancers identifies distinct breast cancer lineages. Nat Commun. (2017) 8:1-14. doi: 10.1038/s41467-017-00510-x

13. Joo JE, Dowty JG, Milne RL, Wong EM, Dugué P-A, English D, et al. Heritable DNA methylation marks associated with susceptibility to breast cancer. Nat Commun. (2018) 9:1-12. doi: 10.1038/s41467-018-03058-6

14. Jiang Z, Lai Y, Beaver JM, Tsegay PS, Zhao M-L, Horton JK, et al. Oxidative DNA damage modulates DNA methylation pattern in human breast cancer 1 (BRCA1) gene via the crosstalk between DNA polymerase $\beta$ and a de novo DNA methyltransferase. Cells. (2020) 9:225. doi: 10.3390/cells9010225

15. Collin LJ, McCullough LE, Conway K, White AJ, Xu X, Cho YH, et al. Reproductive characteristics modify the association between global DNA methylation and breast cancer risk in a population-based sample of women. PLoS ONE. (2019) 14:e0210884. doi: 10.1371/journal.pone.0210884 
16. Yang Y, Cai Q, Shu X, Wu L, Li B, Guo X, et al. DNA methylation quantitative trait loci and breast cancer risk: data from nearly 230,000 women of European descent. AACR. (2018) 78:Abstract nr 5314. doi: 10.1158/1538-7445.AM2018-5314

17. Tang Q, Cheng J, Cao X, Surowy H, Burwinkel B. Blood-based DNA methylation as biomarker for breast cancer: a systematic review. Clin Epigenetics. (2016) 8:115. doi: 10.1186/s13148-016-0282-6

18. Sandoval J, Heyn H, Moran S, Serra-Musach J, Pujana MA, Bibikova M, et al. Validation of a DNA methylation microarray for 450,000 CpG sites in the human genome. Epigenetics. (2011) 6:692-702. doi: 10.4161/epi.6.6.16196

19. Jeschke J, Bizet M, Desmedt C, Calonne E, Dedeurwaerder S, Garaud $\mathrm{S}$, et al. DNA methylation-based immune response signature improves patient diagnosis in multiple cancers. J Clin Invest. (2017) 127:3090-102. doi: 10.1172/JCI91095

20. Tian Y, Morris TJ, Webster AP, Yang Z, Beck S, Feber A, et al. ChAMP: updated methylation analysis pipeline for Illumina BeadChips. Bioinformatics. (2017) 33:3982-4. doi: 10.1093/bioinformatics/btx513

21. Zhou W, Laird PW, Shen H. Comprehensive characterization, annotation and innovative use of Infinium DNA methylation BeadChip probes. Nucleic Acids Res. (2017) 45:e22. doi: 10.1093/nar/gkw967

22. Zhou Y, Zhou B, Pache L, Chang M, Khodabakhshi AH, Tanaseichuk O, et al. Metascape provides a biologist-oriented resource for the analysis of systemslevel datasets. Nat Commun. (2019) 10:1523. doi: 10.1038/s41467-019-09234-6

23. Yang $H$, Wang $K$. Genomic variant annotation and prioritization with ANNOVAR and wANNOVAR. Nat Protoc. (2015) 10:1556-66. doi: 10.1038/nprot.2015.105

24. Kamburov A, Stelzl U, Lehrach H, Herwig R. The ConsensusPathDB interaction database: 2013 update. Nucleic Acids Res. (2013) 41:D793-800. doi: $10.1093 /$ nar/gks1055

25. Ducasse M, Brown MA. Epigenetic aberrations and cancer. Mol Cancer. (2006) 5:60. doi: 10.1186/1476-4598-5-60

26. Novak P, Jensen T, Oshiro MM, Watts GS, Kim CJ, Futscher BW. Agglomerative epigenetic aberrations are a common event in human breast cancer. Cancer Res. (2008) 68:8616-25. doi: 10.1158/0008-5472.CAN-08-1419

27. Gyorffy B, Bottai G, Fleischer T, Munkácsy G, Budczies J, Paladini L, et al. Aberrant DNA methylation impacts gene expression and prognosis in breast cancer subtypes. Int J Cancer. (2016) 138:87-97. doi: 10.1002/ijc.29684

28. Downs BM, Mercado-Rodriguez C, Cimino-Mathews A, Chen C, Yuan JP, Van Den Berg E, et al. DNA methylation markers for breast cancer detection in the developing world. Clin Cancer Res. (2019) 25:6357-67. doi: 10.1158/1078-0432.CCR-18-3277

29. Fackler MJ, Cho S, Cope L, Gabrielson E, Visvanathan K, Wilsbach $\mathrm{K}$, et al. DNA methylation markers predict recurrence-free interval in triple-negative breast cancer. NPJ Breast Cancer. (2020) 6:1-6. doi: 10.1038/s41523-020-0145-3

30. Hsu P-C, Kadlubar SA, Siegel ER, Rogers LJ, Todorova VK, Su LJ, et al. Genome-wide DNA methylation signatures to predict pathologic complete response from combined neoadjuvant chemotherapy with bevacizumab in breast cancer. PLoS ONE. (2020) 15:e0230248. doi: 10.1371/journal.pone.0230248

31. Ren Y, Jiang Y, Zuo W, Xu X, Jin X, Ma D, et al. Abstract P208-33: a novel seven-gene signature predicts prognosis in early-stage triple-negative breast cancer. AACR. (2019) 79:Abstract nr P2-08-33. doi: 10.1158/1538-7445.SABCS18-P2-08-33
32. Wang Q, Li C, Tang P, Ji R, Chen S, Wen J. A minimal IncRNAmRNA signature predicts sensitivity to neoadjuvant chemotherapy in triple-negative breast cancer. Cell Physiol Biochem. (2018) 48:2539-48. doi: 10.1159/000492698

33. Jiang Y-Z, Liu Y-R, Xu X-E, Jin X, Hu X, Yu K-D, et al. Transcriptome analysis of triple-negative breast cancer reveals an integrated mRNA-lncRNA signature with predictive and prognostic value. Cancer Res. (2016) 76:210514. doi: 10.1158/0008-5472.CAN-15-3284

34. Liu Y-R, Jiang Y-Z, Xu X-E, Hu X, Yu K-D, Shao Z-M. Comprehensive transcriptome profiling reveals multigene signatures in triplenegative breast cancer. Clin Cancer Res. (2016) 22:1653-62. doi: 10.1158/1078-0432.CCR-15-1555

35. Fan C-N, Ma L, Liu N. Systematic analysis of lncRNA-miRNA-mRNA competing endogenous RNA network identifies four-lncRNA signature as a prognostic biomarker for breast cancer. J Transl Med. (2018) 16:264. doi: 10.1186/s12967-018-1640-2

36. Terry MB, McDonald JA, Wu HC, Eng S, Santella RM. Epigenetic biomarkers of breast cancer risk: across the breast cancer prevention continuum. In: Stearns V, editor. Novel Biomarkers in the Continuum of Breast Cancer. New York, NY: Springer (2016). p. 33-68.

37. Zhang Y, Wilson R, Heiss J, Breitling LP, Saum K-U, Schöttker B, et al. DNA methylation signatures in peripheral blood strongly predict all-cause mortality. Nat Commun. (2017) 8:1-11. doi: 10.1038/ncomms14617

38. Lian Z-Q, Wang Q, Li W-P, Zhang A-Q, Wu L. Screening of significantly hypermethylated genes in breast cancer using microarray-based methylatedCpG island recovery assay and identification of their expression levels. Int J Oncol. (2012) 41:629-38. doi: 10.3892/ijo.2012.1464

39. Lindqvist BM, Wingren S, Motlagh PB, Nilsson TK. Whole genome DNA methylation signature of HER2-positive breast cancer. Epigenetics. (2014) 9:1149-62. doi: 10.4161/epi.29632

40. Hauptman N, Skok DJ, Spasovska E, Boštjančič E, Glavač D. Genes CEP55, FOXD3, FOXF2, GNAO1, GRIA4, and KCNA5 as potential diagnostic biomarkers in colorectal cancer. BMC Med Genomics. (2019) 12:54. doi: 10.1186/s12920-019-0501-Z

41. Wei Y, Wang X, Zhang Z, Xie M, Li Y, Cao H, et al. Role of polymorphisms of FAM13A, PHLDB1, and CYP24A1 in breast cancer risk. Curr Mol Med. (2019) 19:579-88. doi: 10.2174/15665240196661906191 25109

42. Ziółkowska-Suchanek I, Mosor M, Podralska M, Izykowska K, Gabryel P, Dyszkiewicz W, et al. FAM13A as a novel hypoxia-induced gene in non-small cell lung cancer. J Cancer. (2017) 8:3933. doi: 10.7150/jca. 20342

Conflict of Interest: The authors declare that the research was conducted in the absence of any commercial or financial relationships that could be construed as a potential conflict of interest.

Copyright (C) 2020 Guo, Mao, Qiao, Chen and Jin. This is an open-access article distributed under the terms of the Creative Commons Attribution License (CC BY). The use, distribution or reproduction in other forums is permitted, provided the original author(s) and the copyright owner(s) are credited and that the original publication in this journal is cited, in accordance with accepted academic practice. No use, distribution or reproduction is permitted which does not comply with these terms. 